\title{
Colloidal-structured metallic micro-grids: high performance transparent electrodes in the red and infrared range
}

\author{
Giacomo Torrisi*1,3, João S. Luis², Olalla Sanchez-Sobrado ${ }^{2}$, Rosario Raciti ${ }^{1,3}$, Manuel J. Mendes ${ }^{2}$, \\ Hugo Aguas ${ }^{2}$, Elvira Fortunato ${ }^{2}$, Rodrigo Martins ${ }^{2}$, Antonio Terrasi ${ }^{1,3}$ \\ 1) Università degli Studi di Catania, Department of Physics and Astronomy, Via S. Sofia 64- \\ 95123, Catania (Italy) \\ 2) i3N/CENIMAT, Department of Materials Science, Faculty of Science and Technology, \\ Universidade NOVA de Lisboa and CEMOP/UNINOVA, Campus de Caparica, 2829-516 \\ Caparica, Portugal \\ 3) IMM-CNR, Via S. Sofia, 64 - 95123 Catania (Italy) \\ *corresponding author: giacomo.torrisi@ct.infn.it
}

\begin{abstract}
One of the most promising approaches to produce industrial compatible Transparent Conducting Materials (TCMs) with excellent characteristics is the fabrication of TCO/metal/TCO multilayers. In this article, we report on the electro-optical properties of a novel high-performing TCO/metal/TCO structure in which the intra-layer is a micro-structured metallic grid instead of a continuous thin film. The grid is obtained by evaporation of Ag through a mask of polystyrene colloidal micro-spheres deposited by the Langmuir-Blodgett method and partially dry-etched in plasma. IZO/Ag grid/IZO structures with different thicknesses and mesh dimensions have been fabricated, exhibiting excellent electrical characteristics (sheet resistance below $10 \Omega / \square$ ) and particularly high optical transmittance in the near-infrared spectral region as compared to planar (unstructured) TCM multilayers. Numerical simulations were also used to highlight the role of the Ag mesh parameters on the electrical properties.
\end{abstract}

\section{Introduction}

The increasing interest in developing high-performance and low-cost optoelectronic devices has led to the enhancement of research activities on transparent conductive oxides (TCO) and, more generally, transparent conductive materials (TCMs) [1]. These have allowed a real revolution of optoelectronic devices (solar cells, touch screens, smart windows, LEDs) which are more and more low-cost and high performing [2]-[6]. Amorphous TCOs, for example, are intensively investigated because of their high 
transparency in a wide range (not only in the visible), good electrical conductivity, higher flexibility and low cost [7], [8]. In the specific field of photovoltaics, bifacial solar cells [9] require improved TCMs to optimize the junction properties and to maximize the collection of light from the back side of the cell. Also, there is now an increasing interest in four-terminal mechanically-stacked tandem solar cells, such as the Perovskite/Si double-junctions that already showed $\sim 26 \%$ efficiency, requiring TCMs with particularly high near-infrared (NIR) transparency for the two intermediate contacts between the top (Perovskite-based) and bottom (Si-based) sub-cells [10], [11].

Several approaches have been proposed to fabricate TCM alternatives to TCOs, for example, metal nanowires, graphene or hybrid metal-polymer structures [5], [12]-[14]. Although these materials show interesting basic properties, their optoelectronic performances are still below the TCMs' state-of-the-art and are far from a reliable integration in the industrial fabrication processes. On the other hand, multilayers made of a nano-thin metallic film (from 6 to $20 \mathrm{~nm}$ ) sandwiched in between two TCO layers have demonstrated excellent electrical properties $\left(\mathrm{R}_{\mathrm{sh}}=7.0 \Omega / \square\right)$ and good optical transparency in the visible range $(\mathrm{T} \% \sim 82 \%)$ [15]. For this reason, the hybrid $\mathrm{TCO} / \mathrm{metal} / \mathrm{TCO}$ structure has been extensively investigated and proposed as a valid industrially-compatible TCM for several applications [16]-[19]. In this article, we go further with respect to standard TCO/metal/TCO multilayers by presenting results on the fabrication and characterization of a new type of TCM having, as metal intralayer, a micro-structured Ag grid instead of a continuous thin film. This enables more degrees of freedom in the TCM architecture, allowing to keep the good electrical properties while increasing the optical transparency, mainly in the red-NIR spectral region [20]-[24]. Moreover, this is achieved by using an innovative soft fabrication method (colloidal lithography) which allows low-cost but high-precision micro-patterning throughout indefinitely large surface areas [25]. Although not studied in the present work, the grid is expected to also enable higher flexibility of the TCM in bending processes, relative to planar (unstructured) multilayers [26]. We focused our studies on IZO/Ag grid/IZO by varying three main structural parameters of the Ag grid: thickness and line width of the mesh, as well as the percentage of the uncovered area.

\section{Experimental Methods}

Fabrication: The TCMs studied in this paper consist in a multilayer of IZO/Ag grid/IZO deposited on glass substrates. The bottom and top IZO layers were deposited by RF magnetron sputtering using an $\mathrm{In}_{2} \mathrm{O}_{3} / \mathrm{ZnO}$ target (99.99\% purity, 3" diameter) in a mixture of oxygen and argon with partial pressures of $10^{-5}$ and $1.5 \times 10^{-3}$ mbar, respectively. The applied sputtering power was $50 \mathrm{~W}$ with a deposition rate 
of $4 \mathrm{~nm} / \mathrm{min}$. After the deposition of the IZO bottom layer ( $30 \mathrm{~nm}$ thick), the Ag micro-grid was patterned via a colloidal lithography (CL) process [27]. In this process, a colloidal suspension of polystyrene (PS) micro-spheres, dispersed in solutions at a concentration of $2.5 \% \mathrm{wt}$ in a mixture of water and ethanol (1:3), was used to form a hexagonal closed-packed monolayer array of spheres by Langmuir-Blodgett wet-coating methodology [28]. Colloidal spheres with an original diameter of $1.6 \mu \mathrm{m}$ or $5 \mu \mathrm{m}$ were used in order to form different grids in terms of open areas and line width of mesh. The final size and shape of the spheres, defining the geometry of the resulting mesh, was tailored by exposure to $\mathrm{O}_{2}$ reactive ion etching (RIE) for different times, from 90 to 240 seconds, with a plasma produced with $90 \mathrm{~W}$. Once the spheres were reduced in size by the etching process, Ag was deposited by e-beam evaporation, with a range of thickness between 10 and $70 \mathrm{~nm}$, leading to the formation of the grid through the spheres which were then totally removed with a bath in toluene for $30 \mathrm{~min}$ (lift-off). The last step was the deposition of the IZO top layer (again $30 \mathrm{~nm}$ thick) over the sample surface. A cartoon of the sample preparation steps and structure is shown in Fig. 1a.

Characterization: The optical measurements were performed with a UV-VIS-NIR scanning spectrophotometer (Perkin Elmer lambda 950), using an integrating sphere to obtain total transmittance $\left(\mathrm{T}_{\mathrm{T}}\right)$ and reflectance $\left(\mathrm{R}_{\mathrm{T}}\right)$ spectra. The spectra were used to confirm the thickness of the layers by fitting with an analytical model, based on the Transfer Matrix formalism, which computes the $\mathrm{T}_{\mathrm{T}}$ and $\mathrm{R}_{\mathrm{T}}$ spectra for an arbitrary number of stacked layers [29]. Surface sample morphology was investigated by field emission scanning electron microscopy (SEM) using a Carl Zeiss AURIGA CrossBeam (FIB-SEM) workstation operating at $5.0 \mathrm{kV}$ in the secondary lens operation mode. The surface roughness and the average height of the meshes were measured with an Asylum Research MFP-3D Standalone atomic force microscope (AFM) operating in AC mode. The images were analyzed by the software Gwyddion, in order to obtain the thickness in each sample. The sheet resistances, $\mathrm{R}_{\mathrm{sh}}$, were measured by the fourelectrode Van der Pauw method [30], using a HL5560 system (Bio-Rad, Hercules).

Finally, the electrical behavior of the TCMs was simulated by a finite element method (FEM) based on COMSOL Multiphysics software in order to calculate the current density through the Ag grid and to highlight the role of the mesh dimensions.

\section{Results and discussion}

The fabrication of Ag grids embedded in IZO was pursuit by using different PS micro-sphere diameters, etching time and Ag thickness. Fig. $1 \mathrm{~b}$ reports the area uncovered by the mesh (i.e. regions that were shadowed by the etched spheres during the Ag deposition) and the average value of the minimum width 
of the Ag channels (i.e. the open space along the direction between the centers of two adjacent spheres) as a function of the plasma etching time $(\mathrm{t})$. Data are reported for the two types of PS spheres used in this work, with different initial diameter $(1.6$ and $5 \mu \mathrm{m})$. The SEM images shown in the inset correspond to the grids obtained with such precursor spheres, forming honeycomb arrays with hexagonal symmetry which is typical of structures produced via colloidal lithography.
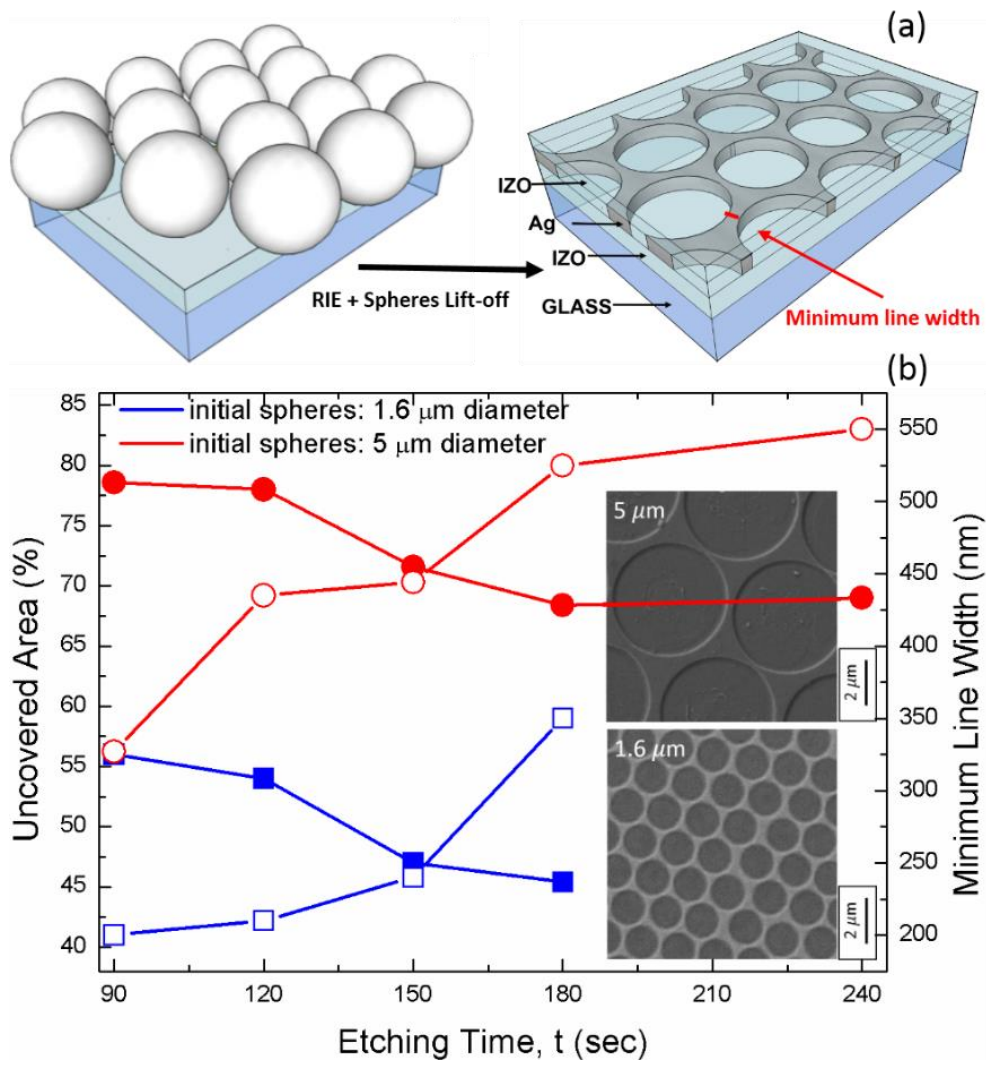

Fig. 1: (a) Schematic of the sample with the PS sphere mask and the final structure of the IZO/Ag grid/IZO. The thickness of the top and bottom IZO layers was kept fixed at $30 \mathrm{~nm}$, for all TCMs analyzed in this work. (b) Area not covered by the mesh, in \% (full symbols), and average minimum Ag channel width (open symbols) vs. etching time. The inset SEM images show Ag grids obtained with spheres of $5 \mu \mathrm{m}$ (top) and $1.6 \mu \mathrm{m}$ (bottom) after $180 \mathrm{~s}$ of etching time.

As expected, increasing the etching time (i.e. decreasing the shadowing spheres' size) produces higher mesh coverage and larger interconnections for both types of samples. The use of the $5 \mu \mathrm{m}$ spheres results in meshes with much larger uncovered area and Ag interconnections, relative to the $1.6 \mu \mathrm{m}$ spheres, which has a strong influence on both optical and electrical properties of the TCMs as discussed below. 
It should be noted that etching times above $200 \mathrm{~s}$ on the small $1.6 \mu \mathrm{m}$ spheres are too aggressive, with the Ag deposition forming almost a continuous film more than a grid. This is the reason why no data at $240 \mathrm{~s}$ of etching is available in Fig. 1 for this sample.

Focusing on the optical analysis, Fig. 2 reports the total transmittance of samples with the same thickness of Ag deposited with or without the PS spheres mask, i.e. respectively with grid or continuous planar Ag films. The most evident result is the significant increase of $\mathrm{T}_{\mathrm{T}}$ in the red and NIR regions of the spectra for both multilayers with grids, as compared to the continuous Ag film. Although $17 \mathrm{~nm}$ of continuous Ag layer still give a transparency of about $70 \%$ at wavelengths around $400-550 \mathrm{~nm}$, there is a tremendous cut-off of the light transmitted above 600-700 nm. On the other hand, when the continuous Ag film is replaced with a grid, $\mathrm{T}_{\mathrm{T}}$ is much higher at any wavelength above $600 \mathrm{~nm}$, lying in between $50 \%$ and 70 $\%$ up to $2000 \mathrm{~nm}$.

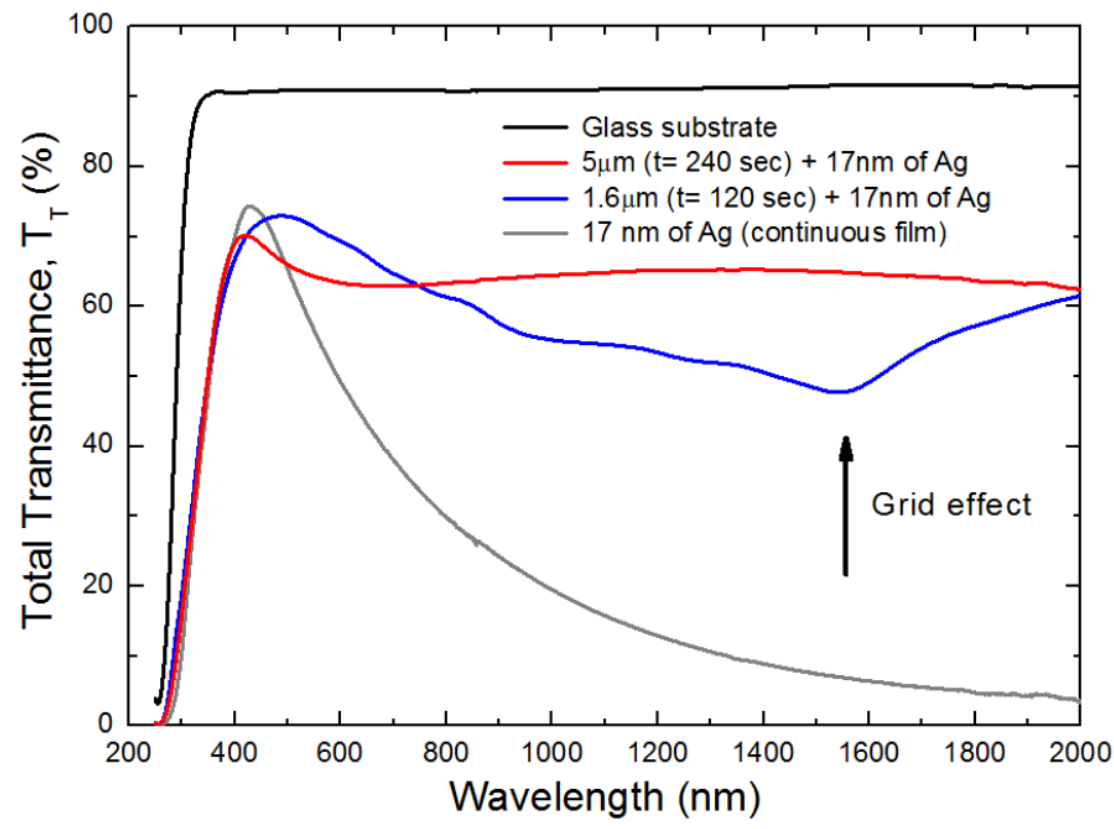

Fig. 2: Transmittance of samples with $17 \mathrm{~nm}$ thick Ag grids (blue and red) and continuous film (grey). The modulation of the blue curve is due to the periodicity of the apertures of the Ag mesh, acting as a diffraction pattern for the incoming light, which results in a minimum in $T_{T}$ at wavelengths close to the pitch (given by the initial sphere diameter).

Another relevant point in the spectrum of the $1.6 \mu \mathrm{m}$ TCM of Fig. 2 is the fact that the pitch of the mesh $(1.6 \mu \mathrm{m})$ is comparable with the wavelengths of the NIR light, therefore producing resonant interference 
and diffraction effects, as demonstrated by the oscillations/modulation of the spectrum, which lead to the minimum around 1500-1700 $\mathrm{nm}$.

The strong enhancement of transparency in the red-NIR region, relative to the continuous film, is thus due to the presence of the grid. As such, we have the possibility of tuning the optical response of the material by controlling the patterning parameters, i.e. the size of the mesh openings and Ag line thickness. This is evidenced by the data reported in Fig. 3, where we show the effects of the plasma etching time and $\mathrm{Ag}$ thickness on the transmittance for the $1.6 \mu \mathrm{m}$ and $5 \mu \mathrm{m}$ spheres. Again, a strong modulation and a lower average value of $\mathrm{T}_{\mathrm{T}}$ is found for samples fabricated with the smaller $1.6 \mu \mathrm{m}$ spheres (Fig. 3a) with respect to the bigger ones (Fig. 3b). A decrease of $\mathrm{T}_{\mathrm{T}}$ with the etching time is observed in all cases, since the etching reduces the size of the spheres which leads to more inter-particle surface exposed to the Ag deposition (larger line width of the resulting mesh).

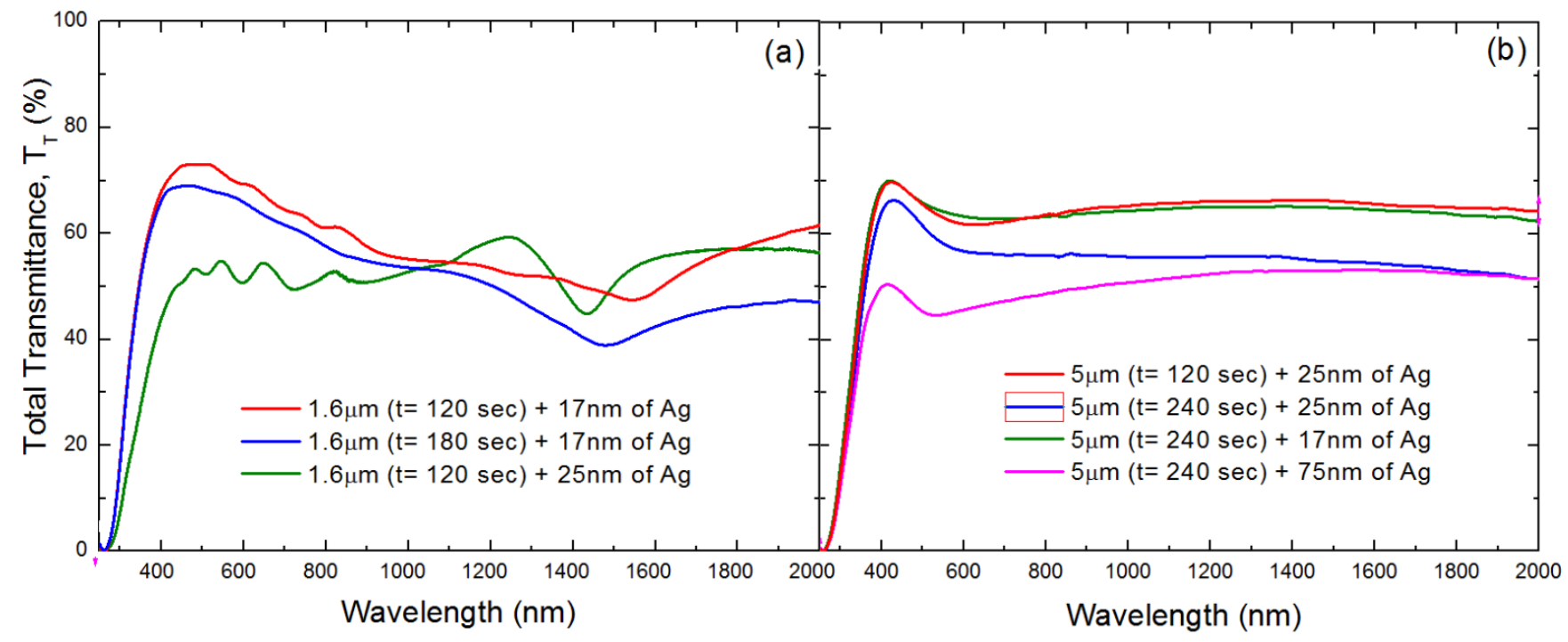

Fig. 3: Effect of the plasma etching times (t) for samples with $1.6 \mu \mathrm{m}(a)$ and $5 \mu \mathrm{m}(\mathrm{b})$ spheres. In the other two panels we report the effect of different Ag thicknesses at a fixed plasma etching time for $1.6 \mu \mathrm{m}(\mathrm{c})$ and $5 \mu \mathrm{m}(d)$ spheres.

Figures 3 also show the effects of different Ag thicknesses while keeping constant the etching time. Apart from the expected reduction of the transmittance with increasing Ag thickness, a major advantageous effect of the grid with respect to the continuous Ag film (shown in Fig. 2) is observed. Specifically, the presence of holes produces a good transparency of the material even for Ag thicknesses ( $25 \mathrm{~nm}$ for the small spheres and $75 \mathrm{~nm}$ for the bigger ones) for which the equivalent continuous film would completely block the transmission of light. 
The optical data reported in Figs. 2 and 3 reveals two important benefits of the grids with respect to continuous films: a much higher transparency in the red-NIR region, and the possibility of tuning the optical response by controlling the structure of the mesh (pitch, line width, thickness).

However, transparent electrodes must join good optical performances to specific electrical properties. In Fig. 4 we report the sheet resistance $\mathrm{R}_{\text {sh }}$ for samples with meshes formed with different initial sphere diameter, etching time and Ag thickness. Beyond the trivial evidence that all samples with a grid have higher $\mathrm{R}_{\mathrm{sh}}$ with respect to those with a continuous planar film, it was found that this value decreases by increasing the etching time and the Ag deposition due to wider and thicker interconnections, respectively.

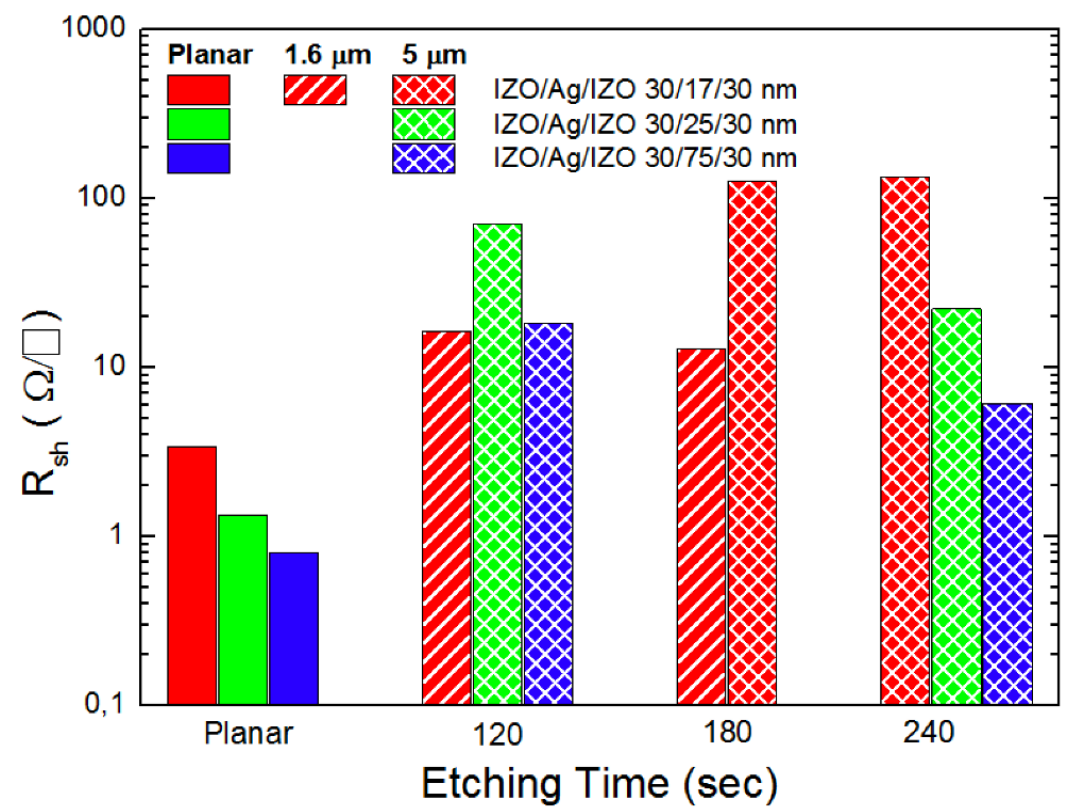

Fig. 4: Sheet resistance of TCMs fabricated with different PS spheres' diameter (1.6 or 5 $\mu \mathrm{m})$, etching time and Ag thickness.

The use of initial small $(1.6 \mu \mathrm{m})$ or large $(5 \mu \mathrm{m})$ spheres produces strong differences in the $\mathrm{R}_{\text {sh }}$ values, being the density of the mesh, i.e. (number of interconnections)/area, much higher for the former with respect to the latter ones. This effect is clearly highlighted, for example, by the large difference of $R_{\text {sh }}(a$ factor of 10) for two samples with the same Ag thickness (17 nm) at $180 \mathrm{~s}$ of etching time.

To provide a better insight on the electrical properties of the mesh structures, we numerically modeled via FEM the current flow through two Ag meshes similar to those reported in the bar plot of Fig. 4 at $180 \mathrm{~s}$ of etching time. In particular, we started by considering a continuous flat Ag film with a sheet resistance of $3 \Omega / \square$. This value of $R_{\text {sh }}$ is attained in the FEM simulation with a $10 \mathrm{~nm}$ thick $\mathrm{Ag}$ film (ideal 
material), while a similar value of $\mathrm{R}_{\mathrm{sh}}$ in Fig. 5 is reported for a sample with $17 \mathrm{~nm}$ of $\mathrm{Ag}$ intra-layer (real material). The effect of mesh-structuring was modeled in the $10 \mathrm{~nm} \mathrm{Ag} \mathrm{film} \mathrm{by} \mathrm{considering} \mathrm{an} \mathrm{hexagonal}$ array of holes, with appropriate size and density in the layer, similar to those obtained after $180 \mathrm{~s}$ of plasma etching with spheres of 1.6 (Fig. 5a) and $5 \mu \mathrm{m}$ (Fig. 5b). Finally, we applied a potential of 1 Volt across the simulated mesh and calculated the current density, $J$ (in $\mathrm{A} / \mathrm{m}^{2}$ ) which is represented in false colors. It is clear how the current density is much lower in the case of $5 \mu \mathrm{m}$ compared to $1.6 \mu \mathrm{m}$, thus yielding a sheet resistance of $124.26 \Omega / \square$ for the former and only $12 \Omega / \square$ for the latter mesh, in precise agreement with the experimental values reported in Fig. 4 at $\mathrm{t}=180 \mathrm{~s}$.
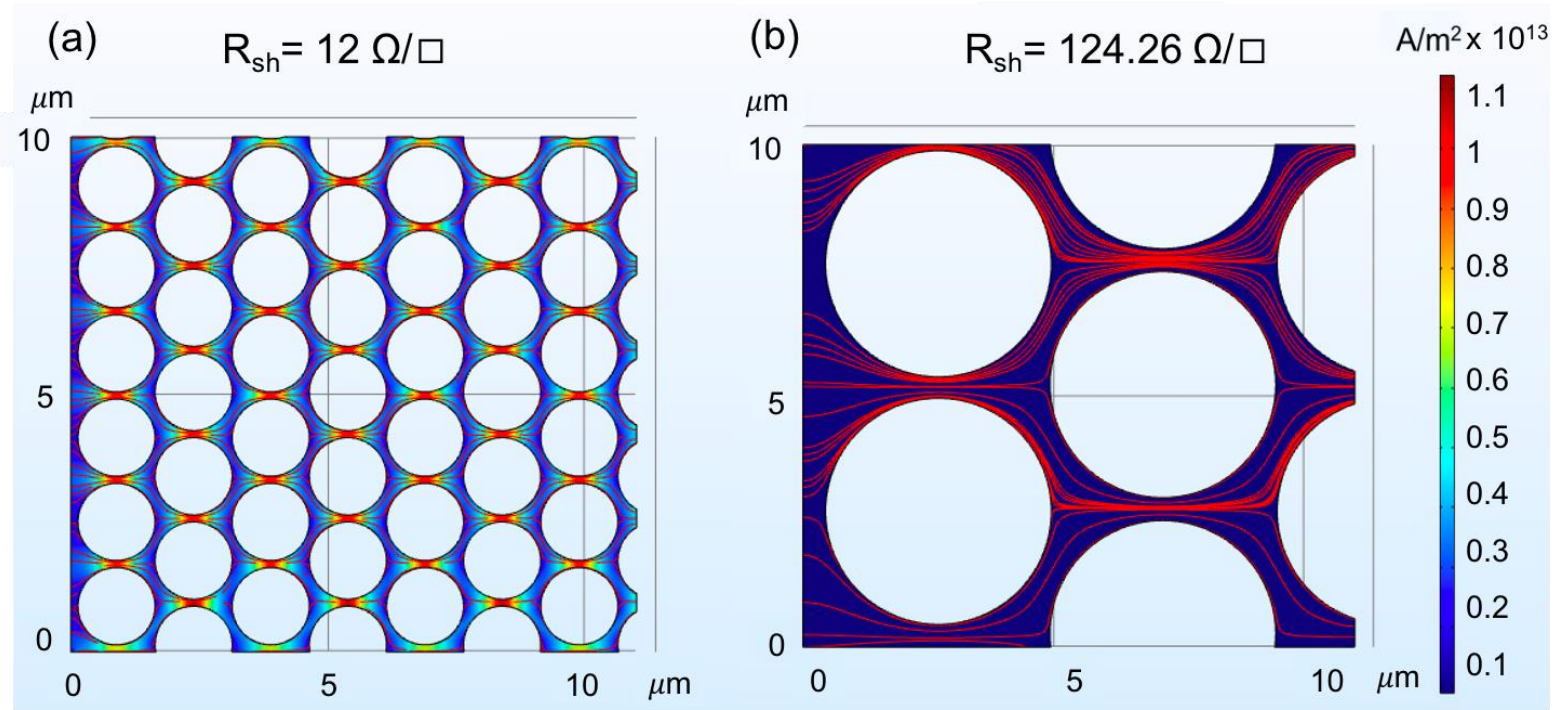

Fig. 5: Simulation of the current density, J, through Ag meshes, $10 \mathrm{~nm}$ thick, similar to those obtained with $180 \mathrm{~s}$ of etching time for the $1.6 \mu \mathrm{m}(\mathrm{a})$ and $5 \mu \mathrm{m}(\mathrm{b})$ spheres. The values of the sheet resistances obtained by the simulation are $12 \Omega / \square$ and $124.26 \Omega \square$ respectively, in excellent agreement with the experimental ones, $12.8 \Omega \square$ and $126 \Omega \square$ reported in Fig. 4. The key role is played by the density of the Ag interconnection lines (i.e. conducting paths) in the grid, which is much higher in the case of smaller spheres.

The electrical properties can be summarized by highlighting the importance of the structural parameters of the Ag mesh on the sheet resistance and current flow in this material. In particular, a mesh with many small apertures and no broken lines can have a better electrical performance with respect to a mesh made of less but larger holes, as seen in the results of Fig. 4. Our simulation clearly shows that this is due to the higher number of paths for the current flow in the former case. This behavior must be taken into account for the overarching optimization of the optical and electrical properties of this type of TCMs. 
The best compromise between optical and electrical properties for TCMs is usually analyzed by means of the so-called Haacke's figure of merit (FoM), i.e. $\mathrm{T}^{10} / \mathrm{R}_{\mathrm{sh}}[31]$. The average $\mathrm{T}$ values used for the FoM of our samples, as reported in Fig. 6, were calculated in the range 700-2000 nm, in order to focus in the red-NIR part of the spectrum in view of the applications (bifacial solar cells, 4-terminal double-junctions, among others) mentioned in the introduction. As a comparison, Fig. 6 also reports the FoM for a stateof-the-art $100 \mathrm{~nm}$ thick ITO film used in commercial photovoltaic devices. It is evident that three of our TCMs attained higher values than the standard ITO layer, and all samples show FoMs that are several orders of magnitudes higher with respect to $\mathrm{TCO} / \mathrm{Ag} / \mathrm{TCO}$ multilayers with continuous $\mathrm{Ag}$ films.

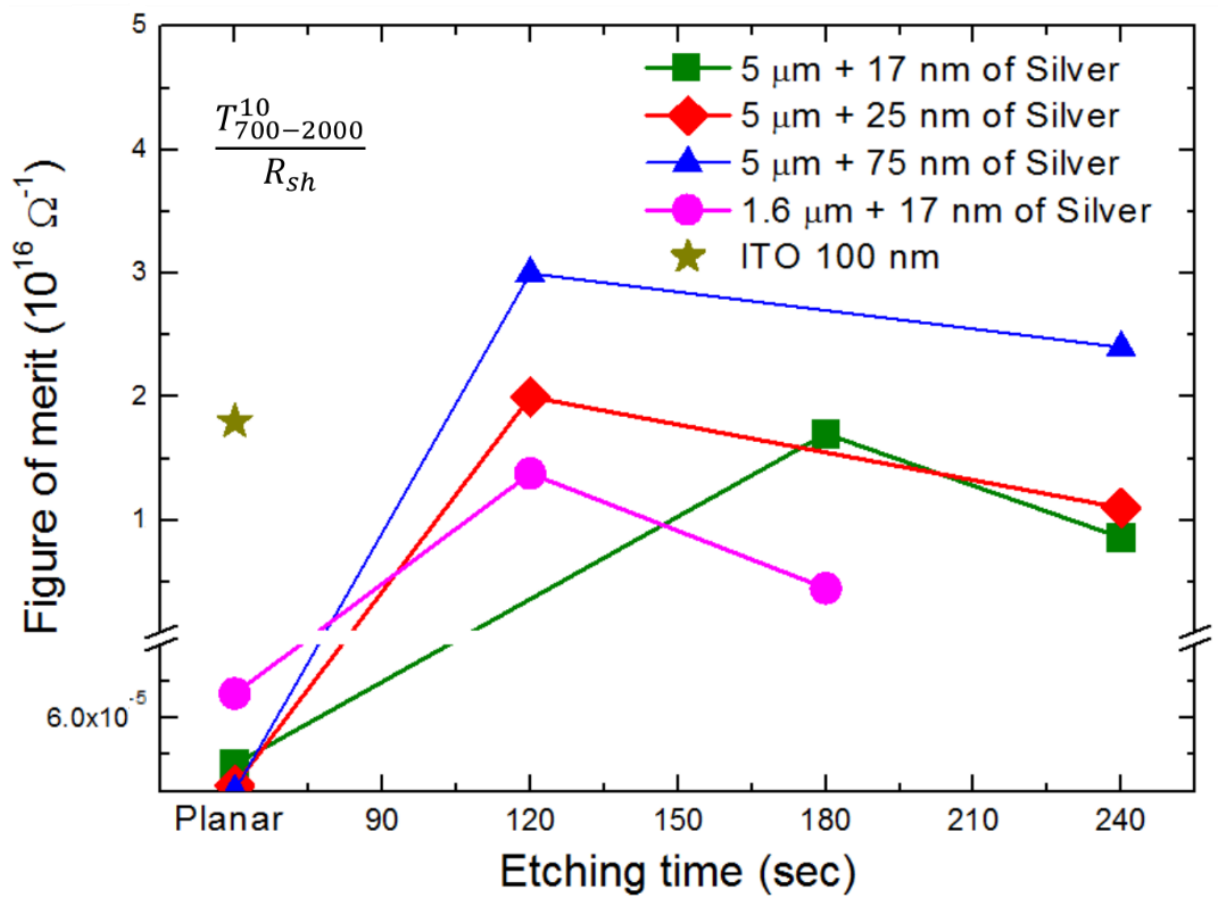

Fig. 6: Haacke's figure of merit for the main TCMs attained in this work, in comparison with a state-of-the-art TCO film made of ITO and with TCMs composed of continuous Ag layers (Planar).

In spite of the fact that the $1.6 \mu \mathrm{m}$ meshes yield much lower $\mathrm{R}_{\mathrm{sh}}$ than the $5 \mu \mathrm{m}$ ones, as shown experimentally (Fig. 4) and via numerical modeling (Fig. 5), it is the latter grids with $75 \mathrm{~nm} \mathrm{Ag}$ thickness that provide the highest FoM values, mainly due to their particularly high red-NIR transmittance (see Fig. 3). 


\section{Conclusions}

In this work we have shown, for the first time to our knowledge, breakthrough physical properties of $\mathrm{TCO} / \mathrm{metal} / \mathrm{TCO}$ multilayer structures in addition to those already known and published for this kind of TCMs. In particular, by replacing the Ag intra-layer film with a micro-grid of the same material, we were able to obtain a much higher transparency in the red-NIR spectral range while enabling highly conductive TCMs. This was achieved by using a mask of etched PS spheres during the Ag deposition, forming IZO/Ag grid/IZO multilayers. The structural parameters of the mesh, i.e. the openings, line width and thickness play a key role to obtain the needed electro-optical response. Meshes made with large (relative to the wavelengths) holes give excellent transmittance values but higher sheet resistance. On the contrary, a mesh with smaller (close to the wavelengths) holes is slightly less transparent but it guarantees a higher electrical current flow. In addition, if a lower sheet resistance is needed in the grids, the thickness of the $\mathrm{Ag}$ material can be increased without a dramatic reduction of the transmitted light. In all cases, thanks to a high percentage of the uncovered surface, the thickness of Ag can be much higher with respect to that of a continuous film, which can also enhance the mechanical robustness of the whole structure. Although not investigated in the present work, the use of a metallic grid instead of a continuous layer can bring important advantages for flexible transparent materials [21]. In conclusion, the use of metallic microgrids as intra-layers in TCO/metal/TCO structures can open new avenues to further improve the electrooptical properties of TCMs. In particular, the remarkably high transparency in the NIR region can be of strategic importance, for instance in smart windows or photovoltaic devices exploiting the low-energy sunlight photons (e.g. for rear contacts of bifacial solar cells or intermediate contacts of multi-terminal multi-junction architectures).

\section{Acknowledgements}

This work was supported by the project PON_206_2 "S5 (Smart Small Scale Solar Systems)", by the project PRN 2014/2020 "BEST-4U”, by FEDER funds through the COMPETE 2020 Programme and National Funds through FCT (Portuguese Foundation for Science and Technology) under the projects UID/CTM/50025/2013, ALTALUZ (PTDC/CTM-ENE/5125/2014), SuPerSolar (PTDC/NANOPT/28430/2017) and TACIT (PTDC/NAN-OPT/28837/2017). O. Sanchez-Sobrado and M. J. Mendes acknowledge funding by FCT through the grants SFRH/BPD/114833/2016 and SFRH/BPD/115566/2016, respectively.

\section{References}


[1] D. R. Sahu, S.-Y. Lin, and J.-L. Huang, " $\mathrm{ZnO} / \mathrm{Ag} / \mathrm{ZnO}$ multilayer films for the application of a very low resistance transparent electrode," Appl. Surf. Sci., vol. 252, no. 20, pp. 7509-7514, Aug. 2006.

[2] Y. F. Lan, W. C. Peng, Y. H. Lo, and J. L. He, "Indium tin oxide films deposited by thermionic-enhanced DC magnetron sputtering on unheated polyethylene terephthalate polymer substrate," Mater. Res. Bull., vol. 44, no. 8, pp. 1760-1764, Aug. 2009.

[3] D. S. Ginley, H. Hosono, and D. C. Paine, Handbook of Transparent Conductors. 2011.

[4] K. Ellmer, "Past achievements and future challenges in the development of optically transparent electrodes," Nat. Photonics, vol. 6, no. 12, pp. 809-817, Dec. 2012.

[5] D. S. Hecht, L. Hu, and G. Irvin, "Emerging Transparent Electrodes Based on Thin Films of Carbon Nanotubes, Graphene, and Metallic Nanostructures," Adv. Mater., vol. 23, no. 13, pp. 1482-1513, Apr. 2011.

[6] A. T. Vicente et al., "Multifunctional cellulose-paper for light harvesting and smart sensing applications," J. Mater. Chem. C, vol. 6, no. 13, pp. 3143-3181, Mar. 2018.

[7] A. Lyubchyk et al., "Influence of post-deposition annealing on electrical and optical properties of ZnObased TCOs deposited at room temperature," Phys. status solidi, vol. 213, no. 9, pp. 2317-2328, Sep. 2016.

[8] S. Marouf et al., "Low-temperature spray-coating of high-performing $\mathrm{ZnO}$ :Al films for transparent electronics,” J. Anal. Appl. Pyrolysis, vol. 127, no. February, pp. 299-308, 2017.

[9] H. Ohta and H. Hosono, "Transparent oxide optoelectronics," Mater. Today, vol. 7, no. 6, pp. 42-51, Jun. 2004.

[10] T. Duong et al., "Rubidium Multication Perovskite with Optimized Bandgap for Perovskite-Silicon Tandem with over 26\% Efficiency," Adv. Energy Mater., vol. 7, no. 14, p. 1700228, Jul. 2017.

[11] D. Shi, Y. Zeng, and W. Shen, "Perovskite/c-Si tandem solar cell with inverted nanopyramids: realizing high efficiency by controllable light trapping," Sci. Rep., vol. 5, no. 1, p. 16504, Dec. 2015.

[12] M. Taguchi et al., "24.7\% Record Efficiency HIT Solar Cell on Thin Silicon Wafer," IEEE J. Photovoltaics, vol. 4, no. 1, pp. 96-99, Jan. 2014.

[13] J.-Y. Lee, S. T. Connor, Y. Cui, and P. Peumans, "Solution-Processed Metal Nanowire Mesh Transparent Electrodes," 2008.

[14] S. Kirchmeyer and K. Reuter, "Scientific importance, properties and growing applications of poly(3,4ethylenedioxythiophene)," J. Mater. Chem., vol. 15, no. 21, p. 2077, May 2005.

[15] I. Crupi, S. Boscarino, V. Strano, S. Mirabella, F. Simone, and A. Terrasi, "Optimization of $\mathrm{ZnO}: \mathrm{Al} / \mathrm{Ag} / \mathrm{ZnO}: \mathrm{Al}$ structures for ultra-thin high-performance transparent conductive electrodes," Thin Solid Films, vol. 520, no. 13, pp. 4432-4435, Apr. 2012.

[16] G. Torrisi, I. Crupi, S. Mirabella, and A. Terrasi, "Robustness and electrical reliability of AZO/Ag/AZO thin film after bending stress,” Sol. Energy Mater. Sol. Cells, vol. 165, pp. 88-93, Jun. 2017.

[17] I. Crupi et al., "Laser irradiation of $\mathrm{ZnO}: \mathrm{Al} / \mathrm{Ag} / \mathrm{ZnO}: \mathrm{Al}$ multilayers for electrical isolation in thin film photovoltaics," Nanoscale Res. Lett., vol. 8, no. 1, 2013.

[18] A. Bingel et al., "AZO/Ag/AZO transparent conductive films: correlation between the structural, electrical, and optical properties and development of an optical model," Opt. Mater. Express, vol. 6, no. 10, p. 3217, Oct. 2016. 
[19] Z. Wang, P. Yi, L. Peng, X. Lai, and J. Ni, "Continuous Fabrication of Highly Conductive and Transparent Ag Mesh Electrodes for Flexible Electronics," IEEE Trans. Nanotechnol., vol. 16, no. 4, pp. 687-694, 2017.

[20] M. J. Mendes et al., "Design of optimized wave-optical spheroidal nanostructures for photonic-enhanced solar cells," Nano Energy, vol. 26, pp. 286-296, Aug. 2016.

[21] W.-K. Kim et al., "Cu Mesh for Flexible Transparent Conductive Electrodes,” Sci. Rep., vol. 5, no. 1, p. 10715, Sep. 2015.

[22] D. S. Ghosh, T. L. Chen, and V. Pruneri, "Ultrathin Cu-Ti bilayer transparent conductors with enhanced figure-of-merit and stability," Appl. Phys. Lett., vol. 96, no. 9, p. 091106, Mar. 2010.

[23] N. Kwon, K. Kim, J. Heo, I. Yi, and I. Chung, "Study on Ag mesh/conductive oxide hybrid transparent electrode for film heaters," Nanotechnology, vol. 25, no. 26, p. 265702, Jul. 2014.

[24] M. M. Adachi, A. J. Labelle, S. M. Thon, X. Lan, S. Hoogland, and E. H. Sargent, "Broadband solar absorption enhancement via periodic nanostructuring of electrodes," Sci. Rep., vol. 3, no. 1, p. 2928, Dec. 2013.

[25] A. J. Morfa, E. M. Akinoglu, J. Subbiah, M. Giersig, and P. Mulvaney, "Transparent metal electrodes from ordered nanosphere arrays," J. Appl. Phys., vol. 114, no. 5, p. 054502, Aug. 2013.

[26] T. Gao, B. Wang, B. Ding, J. Lee, and P. W. Leu, "Uniform and Ordered Copper Nanomeshes by Microsphere Lithography for Transparent Electrodes," Nano Lett., vol. 14, no. 4, pp. 2105-2110, Apr. 2014.

[27] O. Sanchez-Sobrado et al., "Colloidal-lithographed $\mathrm{TiO}_{2}$ photonic nanostructures for solar cell light trapping," J. Mater. Chem. C, vol. 5, no. 27, pp. 6852-6861, Jul. 2017.

[28] R. W. Corkery, "Langmuir-Blodgett (L-B) Multilayer Films," 1997.

[29] M. J. Mendes et al., "Optimal-Enhanced Solar Cell Ultra-thinning with Broadband Nanophotonic Light Capture,” iScience, vol. 3, pp. 238-254, May 2018.

[30] van der Pauw L.J., "A method of measuring specific resistivity and hall effect of discs of arbitrary shape,” Philips Res. Rep., vol. 13, no. 1, pp. 1-9, 1958.

[31] G. Haacke, "New figure of merit for transparent conductors," J. Appl. Phys., vol. 47, no. 9, pp. 40864089, Sep. 1976. 